\title{
Postoperative interstitial hernia as a cause of obscure incisional wound site pain
}

\author{
Andrzej Modrzejewski ${ }^{1}$, Maciej Śmietański ${ }^{2}$ \\ 1Department of General, Vascular and Oncological Surgery and Multiorgan Trauma, District Hospital, Gorzow Wlkp., Poland \\ 2District Hospital, Medical University of Gdansk, Puck, Poland
}

Videosurgery and Other Miniinvasive Techniques 2012; 7 (1): 59-62

DOI: 10.5114/wiitm.2011.23687

\begin{abstract}
An interstitial hernia is one in which the hernia sac is located between the layers of the abdominal wall. The analysis of contemporary literature shows that interstitial hernias are most often seen in children as a type of inguinal hernia and often accompany undescended testis. The hernia sac is usually located between the external-oblique and internaloblique muscles in a lateral-cephalic direction. The authors present 3 cases of interstitial hernia found during laparoscopic exploration of the front abdominal wall done due to incisional wound site pain. No previous diagnosis of hernia was considered in all the cases. Hernias were found as complications of appendectomy and wound healing after radiotherapy of uterine and cervical cancer. In conclusion, in obscure wound site pain, the presence of an interstitial postoperative hernia should be considered as a possible reason for the complaint. Laparoscopic examination of the anterior abdominal wall during adhesiolysis in patients with abdominal pain enables proper diagnosis and treatment.
\end{abstract}

Key words: interstitial hernia, incisional hernia, wound site pain.

\section{Introduction}

An interstitial hernia is one in which the hernia sac is located between the layers of the abdominal wall. It was first described as a variation of inguinal hernia by Bartholin in 1661 [1]. The analysis of contemporary literature shows that interstitial hernias are most often seen in children as a type of inguinal hernia and often accompany undescended testis [1-4]. The hernia sac is usually located between the external-oblique and internal-oblique muscles in a lateral-cephalic direction $[1,4]$. The incidence of interstitial hernias is between $0.08 \%$ [5] and $1.6 \%$ [6]. In one paper, interstitial hernia was also reported as a complication following a Lichtenstein hernioplasty. To date, postoperative evaluations, diagnoses and descriptions of interstitial hernias following other surgical procedures have not been published in medical literature. However, we report 3 cases of interstitial hernia, following difficult laparoscopy procedures, that have been diagnosed in patients with wound site pain.

\section{Case reports}

A 34-year-old male patient was admitted to the hospital for laparoscopic adhesiolysis following an appendectomy performed 4 years previously. The postoperative wound was completely healed; however, the patient complained of continuous pain in the region of the scar (McBurney's approach), and repeated episodes of bowel obstruction were noted. Physical examination (palpation) did not reveal any additional abnormalities. An abdominal ultrasound was 
also inconclusive. Laparoscopy was performed and demonstrated a gap between the transversal and internal-oblique abdominal muscles measuring approximately $5 \mathrm{~cm}$ in diameter. The hernia sac, approximately $6 \mathrm{~cm}$ long, was found sliding between the external and internal oblique muscles. It contained omentum, and two loops of small intestine were bound to the sac's wall. Following this discovery, the patient underwent a mesh closure of the hernia with $5 \mathrm{~cm}$ overlap of the mesh and adjacent muscles (Figure $1 \mathrm{~A}-\mathrm{B}$ ).

A 45-year-old male patient underwent laparoscopy following a paramedian approach appendectomy performed 3 years earlier. The wound healed primarily, but the patient complained of continuous pain in the region of the scar. Abdominal palpation revealed slight tenderness in the region of the scar, with no other abnormalities noted. Abdominal ultrasound showed no other pathological changes. During laparoscopy, an interstitial hernia, with a ring measuring $6 \mathrm{~cm}$ in diameter and a sac penetrating between the transversal and internal-oblique abdominal muscles, was found. The 6-cm-long hernia sac was found sliding in the lateral and cephalic directions. There were several connective tissue bands stretching between the hernia ring edges (Swiss cheese hernia). An open approach was used to liberate the omentum attached to the sac, and polypropylene mesh was stitched to the edges of the muscles with a $5-\mathrm{cm}$ overlap in the sub-lay position (Figure $2 \mathrm{~A}-\mathrm{B}$ ).

A 67-year-old female patient suffered from recurrent episodes of bowel obstruction following radiotherapy for uterine and cervical cancer 18 years previously. On examination there was neo-angiogenesis seen on the skin of the lower abdomen, and palpation revealed hardening of the abdominal wall. Laparoscopy showed a longitudinal gap, $4 \mathrm{~cm}$ long and $1 \mathrm{~cm}$ wide, in the abdominal wall with the hernia sac penetrating into subcutaneous tissue. The hernia orifice was closed via the small skin incision using interrupted stitche (Figure $3 \mathrm{~A}-\mathrm{B}$ ).

\section{Discussion}

Preperitoneal, interstitial and superficial hernias are defined based on anatomical localisation of the hernia sac $[3,4]$. The sac can be located between the peritoneum and transversal fascia (preperitoneal), between the muscle layers (interstitial), or between the fascia of the external-oblique muscle and the skin
A

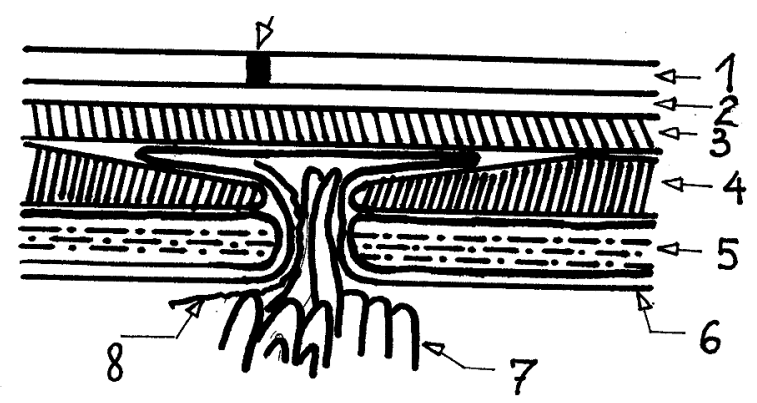

B

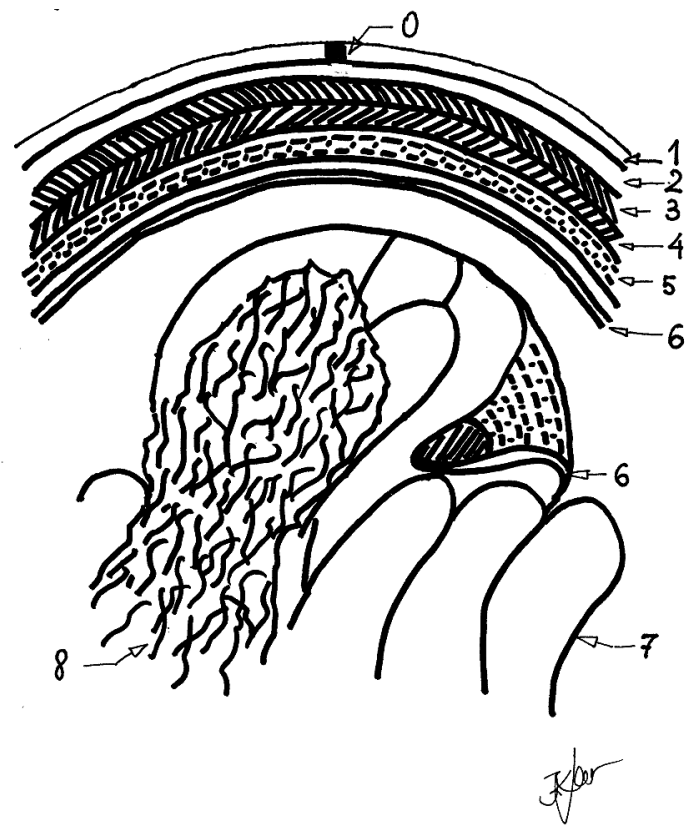

0 - postoperative scar, 1 - skin, 2 - subcutaneous tissue, 3 - external oblique abdominal muscle, 4 - transverse abdominal muscle, 5 - internal oblique abdominal muscle, 6-parietal peritoneum, 7-intestine (loop of the small intestine), 8 -omentum

Figure $1 \mathrm{~A}-\mathrm{B}$. Case 1 
A

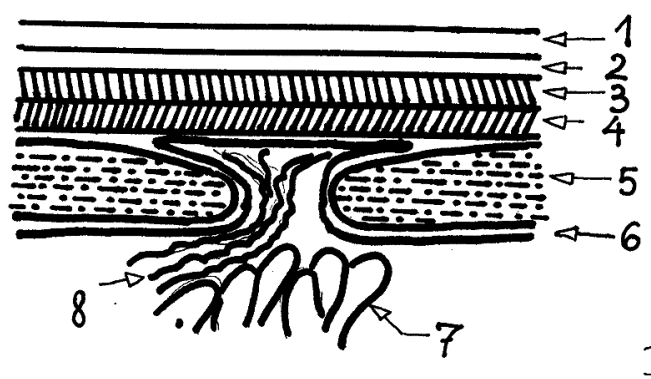

B

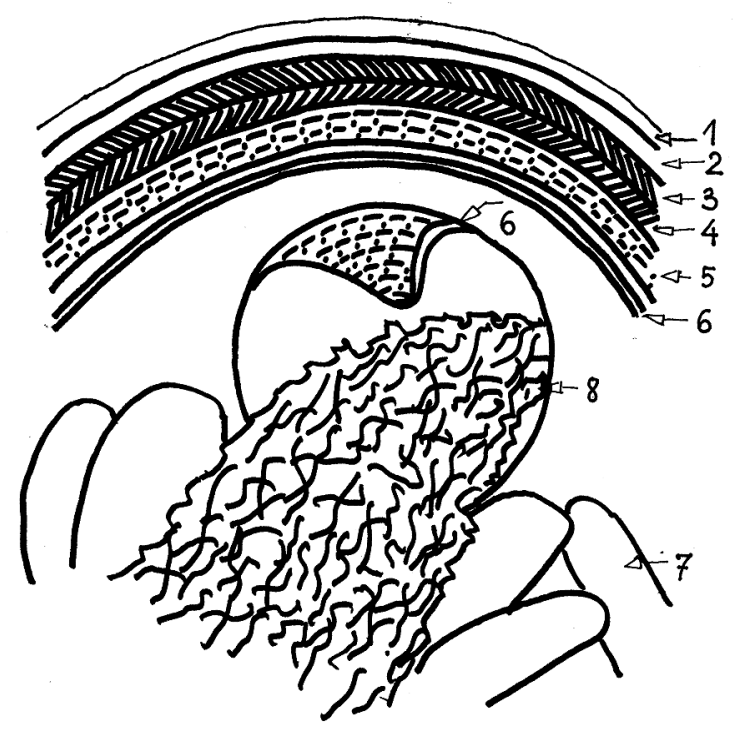

for
A

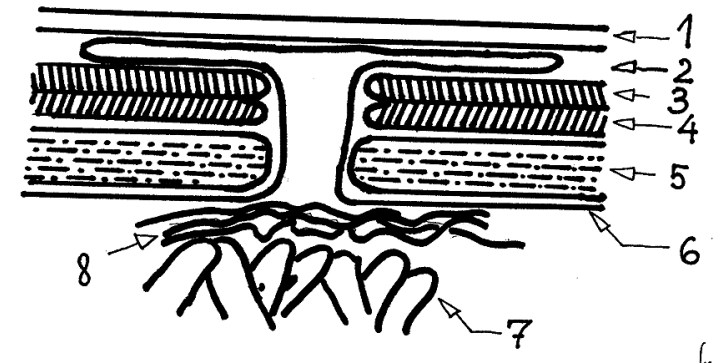
zoer
B

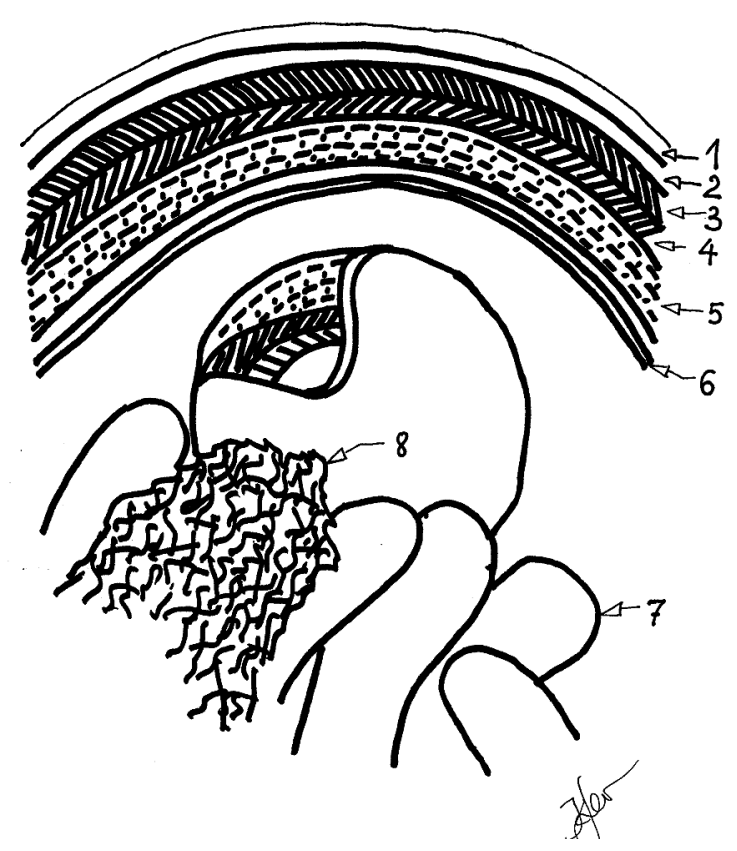


(superficial). Of these types of hernias, the interstitial hernia is the most common [4].

One of the potential mechanisms for developing an interstitial hernia is a narrowing of the external inguinal ring that stops the intestine from descending through the inguinal canal and external inguinal ring [7]. As a result, the hernia sac slides between the abdominal muscles. This theory is supported by the descriptions reported in a number of clinical cases. In one paper, narrowing of the external inguinal ring was so advanced that neither the nerve nor the hernia sac could get through [8]. Other authors present cases of preperitoneal hernia in which the external inguinal ring could not be found during surgery $[9,10]$.

Interstitial hernia can also follow the Lichtenstein hernia repair [11]. Of the three cases just described, only one was diagnosed based on physical examination findings (palpation). The other two diagnoses were based on ultrasound findings. In the aforementioned cases in which an oblique hernia sac was found, one was penetrating in a lumbar direction. Additionally, two cases were treated laparoscopically.

Interstitial hernias make the differential diagnosis of obscure wound site pain more difficult [1], especially when preperitoneal and intramuscular hernia are also considered. This is because the overlying muscle layers make palpation less accurate, and abdominal ultrasound can be useful in these cases. Hernias should be considered along with mobile kidneys, tumours of the caecum and congenital defects of the abdominal musculature.

In our cases, neither palpation nor ultrasound was helpful in establishing a diagnosis. In addition to muscles overlying the hernia sac, the surgical scar also interfered with making the proper diagnosis. Still, ultrasound also does not show other types of hernia in all cases, and in other patients (such as those with groin pain syndrome) the diagnosis is confirmed intraoperatively $[12,13]$. The reason for the development of the described hernias is not explained by the healing process of the external musculature. However, it is possible that tissue infection in the deep layers leads to muscle dehiscence and subsequent hernia formation. Only laparoscopic verification and management of suspected postoperative adhesions led us to the proper diagnosis. Two of the patients described were treated conventionally with mesh implantation. It is well known that such an operation can be performed laparoscopically, while anti-adhesive mesh required for the IPOM (or transabdominal onlay mesh if the hernia is near the inguinal area) procedure is available may require a variable approach [14].

\section{Conclusions}

In cases of obscure wound site pain, the presence of an interstitial postoperative hernia should be considered as a possible reason for the complaint. Laparoscopic examination of the anterior abdominal wall during adhesiolysis in patients with abdominal pain enables proper diagnosis and treatment.

\section{Acknowledgments}

The authors would like to thank Dr Krzysztof Lewandowski for drawing the figures for the manuscript.

\section{References}

1. Múhlbacher L, Hartl H. Interparietale Hernie bei eineiigen Zwillingen. Zbl Chir 1973; 98: 599-601.

2. Langton J. The Bradshaw lecture on the association of inguinal hernia with descent of the testis. Lancet 1900; 2: 1857-64.

3. Lower WE, Hicken NF. Interparietal hernias. Ann Surg 1931; 94 : 1070-87.

4. Koot VCM, de Jong JR, Perre Cl. The interparietal hernia: a rare variant of an inguinal hernia. Eur J Surg 1997; 163: 153-5.

5. Langton J. The Bradshaw lecture on the association of inguinal hernia with descent of the testis. Lancet 1900; 2: 1857-64.

6. Novaro N. Delle ernie inguino-interparietali (inguino-superficiali, inguino-interstiziali, inguino-proerittoneali). Gass Internaz Med 1921; 24: 3-6.

7. Mosetig von Moorhof. Fall von Hernia inguino-proeperitonealis. Wien Med Wochnschr 1885; 35: 257-60.

8. Tillaux P. De la hernie inguino-interstitielle; role du taxis dans cette hernie. Bull Gen de Ther 1871; 71: 209-17.

9. Bramann F. Der processus vaginalis und sein Verhalten bei Störungen des Descensus testiculorum. Arch Klin Chir 1890; 90: 137-68.

10. Butz RV. On properitoneal hernia. Chir Vestnik 1888; 4: 419-22.

11. Read RC, Gilbert AL. Interstitial recurrence, with chronic inguinodynia, after Lichtenstein herniorrhaphy. Hernia 2004; 8: 264-7.

12. Osemek P, Paśnik K, Trojanowski P. Huge, irreducible femoral hernia interpreted as a preperitoneal lipoma in radiological findings. Videosurgery and Other Miniinvasive Techniques 2010; 5: 35-7.

13. Morales-Conde S. Sportsman's hernia: an entity to be defined, diagnosed and treated properly? Videosurgery and Other Miniinvasive Techniques 2009; 4: 32-41.

14. Czudek S, Skrovina M, Adamcik L. Laparoscopic treatment of inguinal hernia - TOM (transabdominal onlay mesh). Videosurgery and Other Miniinvasive Techniques 2009; 4: 16-19. 